\title{
Influence d'un tourbillon océanique sur l'atmosphère
}

\author{
Denis Bourras $^{(1)}$, Guy Caniaux ${ }^{(2)}$, Hervé Giordani ${ }^{(2)}$ et Gilles Reverdin ${ }^{(3)}$ \\ (1) CNRS-IPSL - Centre d'études des environnements terrestre et planétaires - \\ Centre technologique universitaire de Vélizy - 10-12 rue de l'Europe - 78140 Vélizy \\ denis.bourras@cetp.ipsl.fr \\ (2) Météo-France \\ Centre national de recherches météorologiques (CNRM) - Toulouse \\ (3) CNRS - Laboratoire d'océanographie et du climat (Locean) - Paris
}

\section{Résumé}

Lors d'une campagne océanographique récente, on a mis en évidence l'influence sur la couche limite atmosphérique d'une zone tourbillonnaire dans l'océan, restée quasi stationnaire pendant trois mois. Cette zone, d'un diamètre de 100 kilomètres environ, comportait des températures légèrement plus basses que ses alentours. La combinaison des observations et d'une simulation numérique conclut à la présence de circulations atmosphériques fermées dans le plan vertical, en fonction de l'existence ou non du tourbillon froid dans l'océan. Cette étude de cas montre qu'un tourbillon faiblement énergétique a un impact sur la dynamique atmosphérique à une échelle de plusieurs mois, avec des conséquences potentielles sur la variabilité du climat.

\section{Abstract}

\section{Influence of an oceanic vortex on} the atmosphere

A recent oceanic campaign has shown the influence on the atmospheric boundary layer of an oceanic eddy that was quasi-stationary over three months. This area was about $100 \mathrm{~km}$ in diameter and slightly colder than the surroundings. The combination of the observations and a numerical simulation showed that the presence of closed atmospheric circulations in the vertical depended on the existence of the cold eddy in the ocean. This study shows that a weak vortex can have an impact on atmospheric dynamics on a time-scale of months, with potential consequences on climate variability.

\section{$\mathrm{L}$} 'océan et l'atmosphère s'influencent mutuellement. L'étude de ces interactions (interactions océanatmosphère, encadré page 31) est importante pour notre compréhension de la variabilité du climat, et, à plus courte échelle de temps, pour améliorer les prévisions météorologiques et océaniques. Dans cet article, nous nous intéressons au cas particulier de l'influence de l'océan sur l'atmosphère.

La température de surface de la mer (TSM) varie d'une région à l'autre, d'un kilomètre à l'autre, et même d'un mètre à l'autre. À l'image des remous de l'eau bouillonnante dans une casserole que l'on chauffe sur le feu, l'atmosphère s'anime en réponse au chauffage par la surface. Dans le cas de l'océan, on s'intéresse à un chauffage différentiel, car il intervient entre deux régions de températures différentes. D'apparence simple, la réponse de l'atmosphère aux hétérogénéités de TSM est néanmoins un type d'interaction océan-atmosphère qui suscite encore de multiples interrogations.

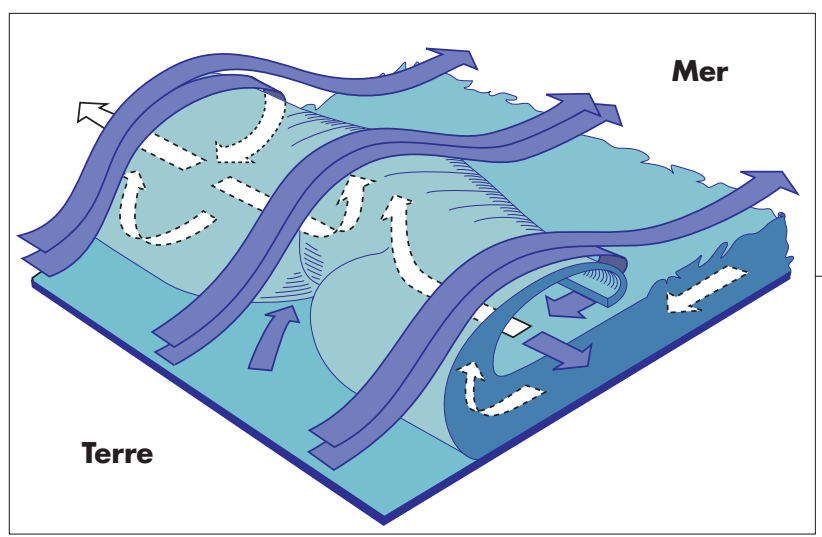

\section{Le chauffage différentiel de l'atmosphère par la surface}

La réponse de l'atmosphère au chauffage par la surface présente des similitudes avec le phénomène de brise de mer, bien connu des véliplanchistes, selon lequel une brise régulière souffle du large dans la journée, et vers le large la nuit. On doit ce phénomène au fait que le soleil chauffe inégalement la mer et la côte. Cette dernière possède une capacité thermique plus faible que celle de l'eau, donc elle se réchauffe plus vite dans la journée, l'inverse se produisant une fois la nuit tombée. Durant le jour, au-dessus de la plage, l'air s'élève car il est chauffé par le sable. Un phénomène de convergence s'ensuit, entraînant l'air du large vers la plage. Le mouvement est complété par un retour du vent vers le large en altitude, et une subsidence au large (Simpson, 1994). Cela crée finalement une cellule de vent agéostrophique, c'est-à-dire une circulation locale du vent uniquement produite par le chauffage différentiel par la surface, encore appelée circulation secondaire (figure 1).

Figure 1 - Circulation atmosphérique de brise de mer. Une circulation s'établit dans le plan vertical, de la mer vers la côte en surface, et du continent vers le large en altitude. (D'après Simpson, 1994) 
De manière analogue, des circulations secondaires sont produites en réponse aux hétérogénéités de TSM. On peut donc rencontrer des vents locaux en pleine mer. Ce phénomène a été observé plus d'une fois au-dessus de la mer, lors de campagnes, ou à partir d'études statistiques reposant sur des champs de TSM, de vent et d'autres variables observés ou obtenus par télédétection spatiale.

\section{Circulations secondaires}

Le contraste thermique est très intense dans le cas de la brise de mer. Il peut atteindre $20{ }^{\circ} \mathrm{C}$ de part et d'autre du front de mer, ce qui constitue un moteur efficace pour alimenter une circulation secondaire. En pleine mer, en revanche, les gradients thermiques sont souvent moins élevés.

Les zones de forts gradients de TSM se rencontrent aux abords des courants marins chauds, comme le Gulf Stream ou le Kuroshio, ou dans les zones de remontée d'eau froide (upwellings). Dans ces régions, les gradients atteignent 10 à 20 degrés sur une distance de 100 kilomètres. Au voisinage du Gulf Stream et dans le Pacifique équatorial, Warner et al. (1990) et Hashizume et al. (2002) ont mis en évidence que l'atmosphère était perturbée sur toute sa hauteur. Ils ont observé des variations de hauteur de couche limite corrélées avec celles de la TSM, indiquant la présence de circulations secondaires.

Mais dans la plupart des océans, les gradients sont très faibles, 1 à $3{ }^{\circ} \mathrm{C}$ sur une distance de $100 \mathrm{~km}$. Dans le cadre de la campagne Semaphore (Structure des échanges mer-atmosphère ; propriétés des hétérogénéités océaniques : recherche expérimentale ; Eymard et al., 1996 ; Kwon et al., 1998 ; Giordani et al., 1998), au large des Açores, Giordani et Planton (2000) ont simulé la réponse de l'atmosphère à un front de TSM de $2{ }^{\circ} \mathrm{C}$ pour $100 \mathrm{~km}$, pendant trois jours, à l'aide d'un modèle météorologique. Leurs analyses montrent clairement la présence de circulations secondaires, de surcroît concentrées sur le premier quart de la hauteur de la couche limite, en raison du faible gradient de TSM. Dans ce cas, les circulations forment ce que l'on appelle une couche limite interne. Rogers (1989) a aussi observé une couche limite interne lors de Fasinex (Frontal Air-Sea Interaction Experiment ; Stage et Weller, 1985 ; Khalsa et Greenhut, 1989 ; Friehe et al., 1991), près des Bermudes.

\section{Les interactions océan-atmosphère}

À la surface de l'océan, l'air et l'eau échangent chaleur, matière et énergie. De ces échanges, résultent des modifications de la dynamique et des propriétés thermodynamiques des deux milieux, que l'on nomme encore interactions océan-atmosphère. Ces dernières occupent un rôle de premier plan dans de nombreux travaux en météorologie, océanographie et étude du climat. Rappelons par exemple que les vagues, au centre de nombreuses études, sont principalement formées sous l'action du vent, et sont la manifestation de l'énergie mécanique qui est transmise à l'océan par l'atmosphère. Autre exemple, l'énergie solaire chauffe par rayonnement les eaux superficielles de l'océan. En retour, l'océan chauffe l'atmosphère par convection. Simultanément, l'eau s'évapore, et l'air chaud chargé d'humidité au voisinage de la surface s'élève par poussée d'Archimède. L'océan émet ainsi des quantités d'eau et de chaleur dans l'atmosphère, qui pourront ensuite se condenser sous forme de nuages. C'est aussi par ce processus de chauffage à la base que l'atmosphère se met en mouvement.

Les échanges de chaleur entre atmosphère et océan jouent un rôle important sur la météorologie et le climat. La première raison est l'importance des océans en termes de superficie. D'autre part, l'inertie thermique du système océan est quatre fois supérieure à celle de l'air, et est plus élevée que celle du continent. Cette raison justifie à elle seule le rôle central de l'océan et de ses échanges avec l'atmosphère sur le système climatique. Enfin, il faut aussi comprendre que, contrairement au continent, l'océan est en perpétuel mouvement, et qu'un simple gradient de température ou de salinité en surface ou encore l'action du vent peut faire plonger des masses d'eau, ou bien en faire remonter à la surface après des dizaines d'années. Autrement dit, l'océan peut réserver des surprises en termes de climat, ce qui est moins évident pour le continent - hormis les possibles variations du couvert végétal, de la glace, de la neige ou de l'humidité des sols et du couvert végétal -, parce qu'il est statique aux échelles météorologiques et climatiques.

Les interactions océan-atmosphère se produisent dans une région de plusieurs centaines de mètres à plusieurs kilomètres de haut dans l'atmosphère, la couche limite atmosphérique, et de quelques dizaines à quelques centaines de mètres de profondeur, la couche de mélange océanique. Dans cette zone frontière entre les milieux air et eau, l'écoulement atmosphérique est principalement turbulent. Les tourbillons transportent la chaleur, l'énergie mécanique, et les constituants qui passent de l'air vers l'océan, et réciproquement.

À l'interface, on quantifie les échanges entre océan et atmosphère sous forme de flux de chaleur, d'énergie et de matière.

- Le flux d'énergie mécanique est appelé flux turbulent de quantité de mouvement. C'est le produit du frottement du vent contre la surface de la mer. Il prélève l'énergie à l'atmosphère (donc le vent diminue à mesure que l'on s'approche de la surface) sous forme d'une activité tourbillonnaire dans la couche limite atmosphérique et de vagues en surface.

- Les flux de matière sont par exemple les flux d'aérosols marins, de constituants chimiques comme le dioxyde de carbone ou les diméthyl-sulfates dont le rôle sur le climat est bien connu, mais on entend aussi par ce terme le flux d'eau qui passe de l'océan vers l'air s'il s'agit d'évaporation, ou le contraire s'il s'agit de condensation ou de pluie. - Les flux de chaleur peuvent être décomposés en quatre postes : deux flux radiatifs, respectivement dans le visible et l'infrarouge, caractérisant les rayonnements du soleil, de l'atmosphère, des nuages et de l'océan, et deux flux turbulents liés, d'une part, aux échanges de température entre air et eau (le flux de chaleur sensible) et, d'autre part, aux échanges d'humidité (flux de chaleur latente d'évaporation ou de condensation).

À l'échelle de l'ensemble des océans et en moyenne annuelle, les océans reçoivent une quantité de chaleur du soleil avoisinant les $200 \mathrm{~W} \cdot \mathrm{m}^{-2}$ (en fait, plus de $1300 \mathrm{~W} \cdot \mathrm{m}^{-2}$ arrivent au sommet de l'atmosphère, mais la plupart sont arrêtés par la couverture nuageuse et par l'atmosphère), sous forme de flux radiatif dans les longueurs d'ondes visibles. Les océans restituent $150 \mathrm{~W} \cdot \mathrm{m}^{-2}$ à l'atmosphère par évaporation (flux de chaleur latente), $40 \mathrm{~W} \cdot \mathrm{m}^{-2}$ par rayonnement dans l'infrarouge et $10 \mathrm{~W} \cdot \mathrm{m}^{-2}$ sous forme d'échanges de température (flux de chaleur sensible). Ces chiffres ne sont que des ordres de grandeur des différents termes du bilan radiatif à la surface des océans. Par définition, sur une longue durée et à l'échelle du globe, la somme algébrique des différents termes doit être nulle, à moins que l'océan ne se réchauffe, ou se refroidisse.

Enfin, un aspect très important des interactions océan-atmosphère concerne leur rôle dans les modèles numériques d'océan et d'atmosphère. Pour ces modèles, les flux à I'interface sont une condition aux limites indispensable. Dans ce but, on effectue des campagnes de mesure, pendant lesquelles on observe les propriétés de l'océan, de l'atmosphère, et les flux de chaleur et d'énergie entre les deux milieux. Ces observations, combinées avec les approches théoriques, permettent de relier mathématiquement les conditions environnementales aux flux, pour finalement établir ce que l'on nomme une paramétrisation des flux de surface. 
Pris dans leur ensemble, les résultats présentés dans ce paragraphe suggèrent que la hauteur des circulations croît avec l'intensité des gradients de surface, mais cela reste à démontrer et dépend sans doute des échelles de temps et d'espace considérées.

\section{Échelles de temps et d'espace}

Le phénomène de réponse de l'atmosphère au chauffage différentiel par la surface intervient à presque toutes les échelles de temps et d'espace, avec divers niveaux d'intensité. À l'échelle du globe, par exemple, la circulation du vent est essentiellement contrainte par les gradients thermiques en surface, la force de Coriolis et l'orographie. Plus haut, nous avons cité l'exemple de la brise de mer, qui est un effet très local se produisant à échelle de quelques heures. Donc, une très large palette d'échelles de temps et d'espace est impliquée. Il est important de mieux comprendre cet aspect, car, même si l'effet du chauffage par la surface peut être mêlé à l'écoulement synoptique, voire négligeable par rapport à ce dernier, il est possible que la réponse de l'atmosphère soit un déclencheur de phénomènes météorologiques de grande échelle.

Les jeux de données mondiales de vent de surface et de TSM, assemblés depuis les années 1980, ont contribué à mieux cerner le rôle de diverses échelles de temps et d'espace, ainsi que leurs interactions. Les données du Comprehensive Ocean-Atmosphere Dataset, ou Coads, (Woodruff et al., 1987) ont permis à Wallace et al. (1989) de montrer que le vent réagissait aux gradients de TSM à des échelles de temps de vingt jours à six mois, ce qui a été confirmé par des études plus récentes effectuées à partir des produits issus de la télédétection spatiale (Chelton et al., 2001; White et Annis, 2003). Cependant, ces études ont surtout porté sur les régions de forts gradients de TSM, où la relation ventTSM pouvait être clairement mise en évidence. Dans les cas de faibles gradients de TSM, la réponse n'a pas encore été apportée, tout au moins aux échelles saisonnières. L'écoulement dans les couches superficielles de l'océan est essentiellement tourbillonnaire, et la température n'est pas la même d'un tourbillon à l'autre. Bien que les écarts soient faibles, certains d'entre eux peuvent perdurer plusieurs semaines, voire plusieurs mois. Il est donc légitime de se demander si les tourbillons faiblement énergétiques et persistants ont un impact sur le vent, et plus généralement sur les autres propriétés de l'atmosphère.

\section{Processus de génération des circulations}

Il est admis que le chauffage différentiel par la surface provoque un écart de pression atmosphérique entre les côtés chaud et froid d'un front de TSM, ce qui en retour génère une circulation atmosphérique. Depuis Wallace et al. (1989), on sait cependant que l'intensité de la turbulence est aussi un processus candidat pour expliquer la réponse de l'atmosphère. Son principe est assez peu intuitif : l'intensité du mélange turbulent est accrue au-dessus du côté chaud, car l'air, plus chauffé à sa base, y est naturellement plus instable que du côté froid. Cela contribue à renforcer le vent du côté chaud. À la surface, le vent se dirige du côté froid vers le côté chaud du front, et plus de turbulence est synonyme de vent plus intense au-dessus du côté chaud. En résumé, si l'effet turbulent prédomine, les anomalies de TSM sont en phase avec les anomalies d'intensité du vent de surface. En revanche, dans le cas où c'est l'effet de pression qui est prépondérant, les anomalies de TSM et de vent sont déphasées de 90 degrés. Giordani et Planton (2000) ont montré récemment que plusieurs autres processus, d'origine thermique ou dynamique, pouvaient intervenir à parts égales avec les deux précédents. L'étude de Giordani et Planton est toutefois limitée à trois jours de simulations, sur une région de quelques centaines de kilomètres. Il s'agit donc d'une étude de cas et on n'a pas encore examiné le ou les processus prépondérants dans d'autres types de situations météorologiques.

\section{Étude de cas pendant la campagne Pomme}

Nous présentons ci-après l'étude d'un tourbillon faiblement énergétique et son influence sur l'atmosphère. Le tourbillon, plus froid (de l'ordre de $1^{\circ} \mathrm{C}$ ) que son environnement, a été repéré en Atlantique Nord-Est, à environ $500 \mathrm{~km}$ à l'ouest des Canaries, à l'occasion de la campagne Pomme dédiée à l'étude de la subduction des eaux modales (encadré ci-dessous). Observations et simulations nous ont permis d'établir tour à tour que le module du vent décroissait en réponse à la présence du tourbillon froid, que la couche limite atmosphérique était influencée sur toute sa hauteur, que l'atmosphère était perturbée via un effet de turbulence et non de pression.

\section{La campagne Pomme}

Le programme océanographique Pomme (Programme océan multidisciplinaire méso échelle) dans l'Atlantique Nord avait pour objectif de mieux comprendre le rôle de l'océan sur le climat. Dans le cadre de ce programme coordonné par l'Institut national des sciences de l'univers (Insu), les scientifiques du CNRS, du Service hydrographique de la marine (Shom), de I' Institut français de recherche pour l'exploitation de la mer (Ifremer) et de Météo-France ont coopéré.

Les objectifs principaux étaient de comprendre comment les eaux océaniques hivernales de surface s'enfouissent à des profondeurs intermédiaires et comment les mécanismes biologiques et physiques interagissent sur le stockage du carbone dans l'océan. Durant l'hiver, l'océan subit des pertes de chaleur importantes vers l'atmosphère : la couche océanique de surface, directement en contact avec l'atmosphère, mélangée par l'action du vent, s'approfondit pour atteindre quelques centaines de mètres. Au printemps, l'énergie solaire chauffe la partie supérieure de cette couche mélangée hivernale sur quelques dizaines de mètres. Ainsi, la partie inférieure de la couche mélangée hivernale devient isolée de l'atmosphère : la circulation océanique transporte ensuite cette masse d'eau isolée et chargée en carbone sur de grandes distances (plusieurs milliers de kilomètres, à l'échelle de bassins océaniques). Ce processus complexe, appelé processus de "subduction », a lieu en fin d'hiver - début de printemps. C'est à peu près durant la même période que se développe la floraison printanière, accroissement spectaculaire de la biomasse marine (matière organique) sous forme de phytoplancton (petites algues dont la taille est de l'ordre du micron, premier échelon de la chaîne alimentaire marine). La subduction des eaux de surface joue un rôle essentiel dans la régulation du climat et des cycles biogéochimiques (carbone, oxygène, etc.). Elle isole en effet les masses d'eau, et empêche donc les échanges de chaleur et de matière avec l'atmosphère. En outre, elle permet à l'océan de garder en mémoire les conditions climatiques de surface durant plusieurs années. 
L'intérêt de cette étude est d'abord de documenter un cas d'interaction océanatmosphère, les processus mis en jeu, leur observation et les caractéristiques spatio-temporelles du phénomène, en réponse aux questions exposées cidessus. Mais, surtout, il s'agit d'une des premières études traitant de l'impact sur l'atmosphère des tourbillons faiblement énergétiques, que l'on rencontre pourtant un peu partout dans l'océan.

\section{Campagne et données}

La région étudiée à l'occasion de la campagne Pomme s'étend de $35^{\circ} \mathrm{N}$ $15^{\circ} \mathrm{W}$ à $48^{\circ} \mathrm{N}-25^{\circ} \mathrm{W}$ (figure 2). Les données de la campagne ont été collectées lors de la période d'observations intensives (POI), qui a débuté le $1^{\text {er }}$ février 2001 et s'est terminée le 30 avril 2001. Les conditions rencontrées pendant cette période sont caractéristiques du réchauffement de printemps à ces latitudes :

- des vents de $8 \mathrm{~m} . \mathrm{s}^{-1}$;

- une TSM de $14{ }^{\circ} \mathrm{C}$ s'accroissant de $0,9^{\circ} \mathrm{C}$ en trois mois ;

- un flux de chaleur négatif (l'océan perd de la chaleur, donc réchauffe l'atmosphère) en début de $\mathrm{POI}$ et positif (entrant dans l'océan), de $100 \mathrm{~W} \mathrm{Wm}^{-2}$, en fin de période.

La zone Pomme est traversée par une branche de la dérive nord-atlantique. Cependant, elle n'est marquée par aucun contraste thermique violent en surface, contrairement à la région du Gulf Stream, par exemple. On note aussi que les tourbillons océaniques sont moins énergétiques dans la zone Pomme que sur la partie ouest du bassin (Ducet et al., 2000). Paradoxalement, ces caractéristiques rendent la région Pomme intéressante du point de vue des interactions océan-atmosphère, car elle est représentative de la majorité des océans aux latitudes moyennes.

Lors de la campagne, le dispositif instrumental déployé comportait, pour la partie relative à cette étude, une trentaine de bouées dérivantes, mesurant notamment la vitesse du vent, et un navire océanographique de l'Ifremer, L'Atalante, à bord duquel on a mesuré les variables météorologiques, océaniques et les flux de surface.

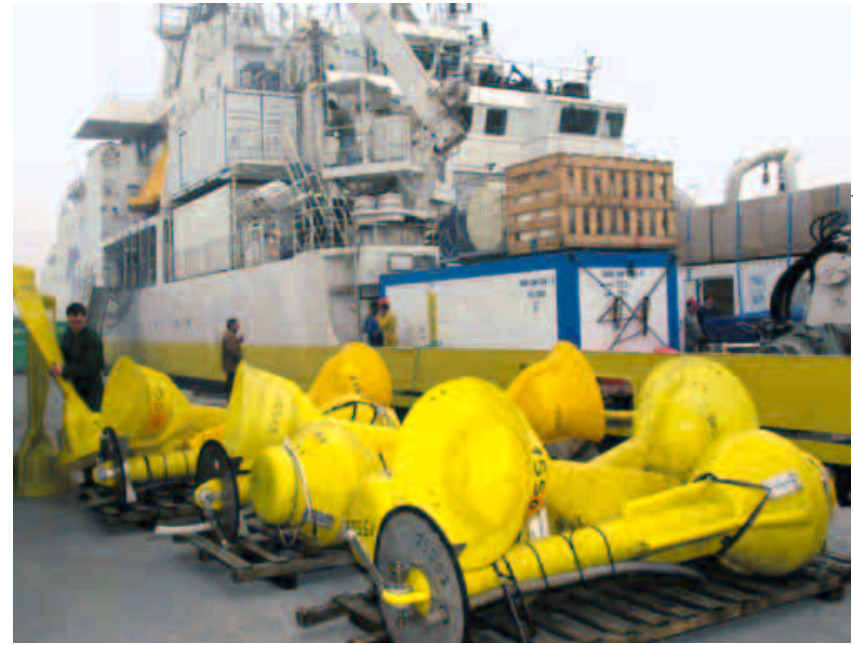

Des bouées Marisonde en instance de départ pour la campagne Pomme. (Photo Météo-France, P. Taburet)

\section{Apport \\ de la télédétection spatiale}

Nous avons complété les mesures de Pomme par des observations spatiales. Celles-ci constituent une aide précieuse pour identifier les interactions océanatmosphère, car elles fournissent des observations avec un bon échantillonnage spatio-temporel de la zone, soit deux fois par jour sur une maille de $30 \mathrm{~km}$ par $30 \mathrm{~km}$ pour les satellites défilants, et toutes les demi-heures à la résolution de $1 \mathrm{~km}$ pour les géostationnaires comme Météosat. Les variables estimées par satellite sont la TSM, le vent et les flux de surface.

Pour estimer la TSM, on utilise souvent les radiomètres infrarouges tels que le Advanced Very High Resolution Radiometer (AVHRR) de la National Oceanic and Atmospheric Administration (NOAA). Ils mesurent la quantité de rayonnement électromagnétique naturellement émise par la surface, qui est fonction de sa température. La TSM est obtenue à $\pm 0,3{ }^{\circ} \mathrm{C}$ près, ce qui est précis (Kilpatrick et al., 2001). L'instrument présente cependant la limite d'être aveugle en présence de nuages. Pour obtenir un échantillonnage complet de la région Pomme, Caniaux et

Figure 2 - Région de l'Atlantique Nord-Est étudiée lors du programme Pomme. Limites en rouge : zone d'étude intensive lors des campagnes océanographiques. Limites en vert : zone couverte par le modèle numérique de circulation océanique régionale Soprane, utilisé au cours de Pomme. Ligne en trait tireté : zone approximative de l'enfouissement dans l'océan intermédiaire vers le sud des masses d'eau de surface provenant du nord. Les flèches bleues représentent les principaux courants marins.

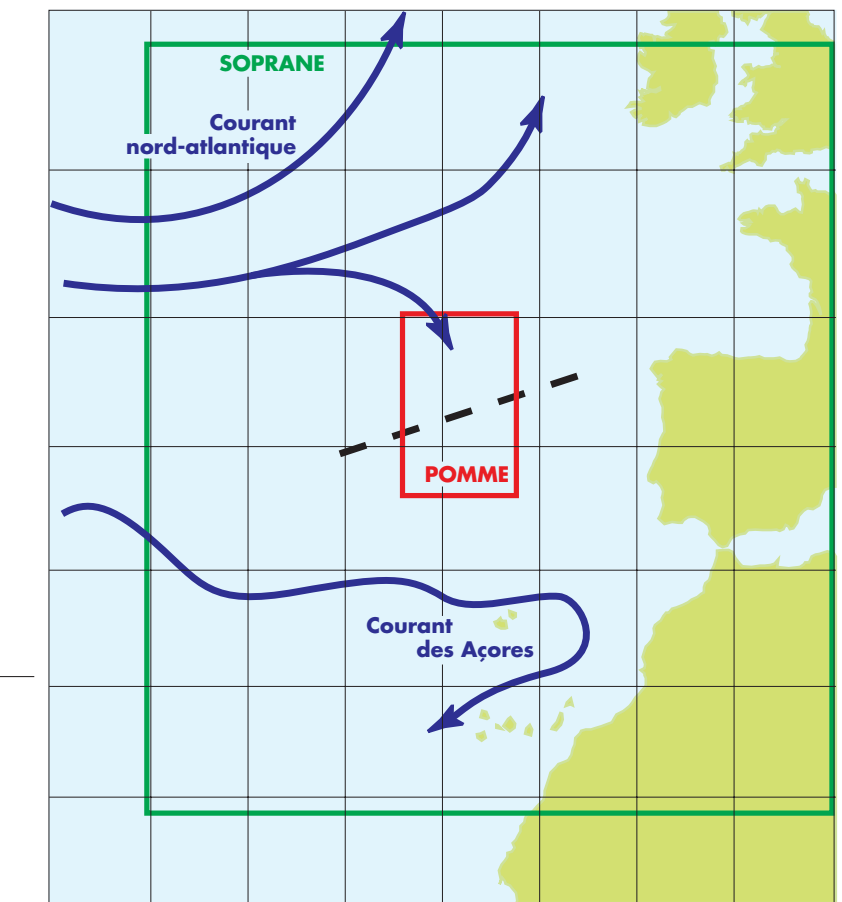

al. (2005) ont interpolé les TSM d'AVHRR et les n o m b r e u s s mesures de TSM obtenues localement pendant la campagne. Ils ont ainsi produit des champs de flux durant toute la POI, toutes les 6 heures à la résolution de 5 kilomètres.

Le module du vent à la surface des océans est un produit fiable de la télédétection spatiale. On le restitue avec une précision de 1 à $2 \mathrm{~m} . \mathrm{s}^{-1}$ pour des observations instantanées. Les instruments spatiaux ne voient pas directement le vent, mais plutôt la déformation de la surface de la mer sous l'action du vent (des vagues capillaires de quelques centimètres de longueur d'onde). On estime donc le vent, soit à partir d'un écho radar modifié par ces capillaires, soit par leur émissivité naturelle. Celle-ci est adaptée aux hyperfréquences, car de longueur d'onde identique. Pour Pomme, nous avons utilisé les deux types d'instruments, le radiomètre passif SSM/I (Spectral Sensor Microwave Imager) de la NOAA et Quikscat, un radar diffusiomètre hyperfréquences. 
On déduit le flux de chaleur à travers l'interface océan-atmosphère d'une combinaison des mesures de Météosat, AVHRR et SSM/I. La précision des flux obtenus n'est que de 30 à $50 \mathrm{~W} / \mathrm{m}^{2}$, soit 30 à $40 \%$ d'erreur. Cependant, ces champs se révèlent souvent utiles pour des études qualitatives, ou dans les cas où la variabilité spatiale importe plus qu'une information absolue en termes de flux (Bourras et al., 2003, 2004).

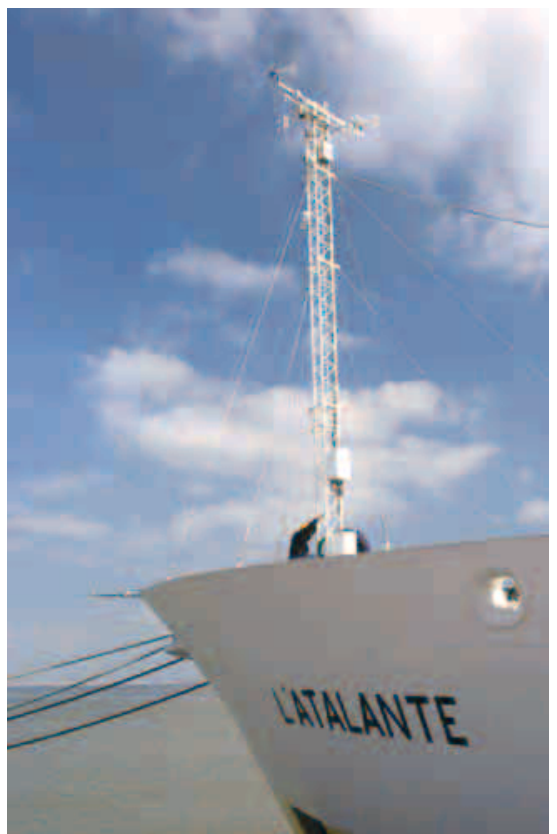

L'Atalante, navire océanographique de l'Ifremer, équipé d'un mât anémométrique et d'une perche de mesure à l'étrave lors de la campagne Pomme. (Photo MétéoFrance, P. Taburet)

\section{Un tourbillon dans l'Atlantique Nord-Est}

La carte des TSM moyennes du

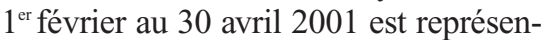
tée dans la figure $3 \mathrm{~b}$. On y découvre une forte activité tourbillonnaire de mésoéchelle, en particulier une zone tourbillonnaire froide circulaire, centrée sur $19,5^{\circ} \mathrm{W}-41,5^{\circ} \mathrm{N}$ et dont le diamètre est de l'ordre de $100 \mathrm{~km}$. Cette zone, appelée tourbillon froid dans la suite, regroupe un ensemble de tourbillons cycloniques persistant à l'échelle de trois mois, et plus froids d'environ $1{ }^{\circ} \mathrm{C}$ par rapport à leur environnement. Pour référence, la figure 3 a montre la TSM issue du modèle opérationnel du Centre européen de prévisions météorologiques à moyen terme (CEPMMT). Cette dernière est beaucoup plus lisse que le produit de Caniaux et al. (2005) et ne comporte pas le tourbillon froid. La figure $3 \mathrm{~d}$ indique la présence d'une structure de flux de chaleur qui se juxtapose parfaitement au tourbillon froid. Cette anomalie semble être liée à un effet de surface, car on l'observe sur les champs de flux de surface, mais pas sur les champs de flux radiatifs descendants, c'est-à-dire les flux solaire et infrarouge, liés notamment à la nébulo-
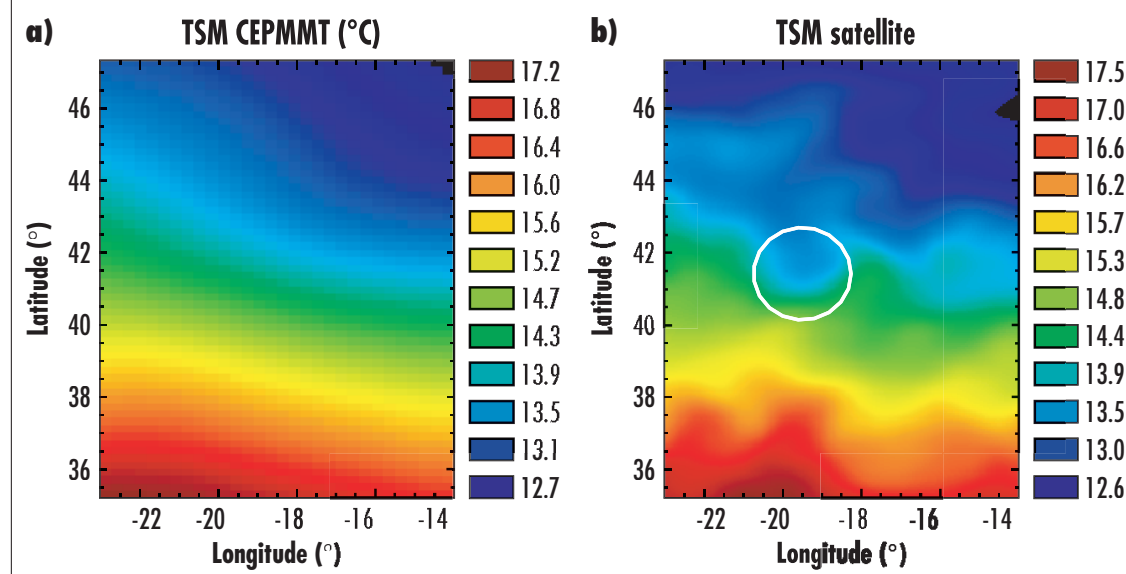

c) Flux de chaleur net - CEPMMI

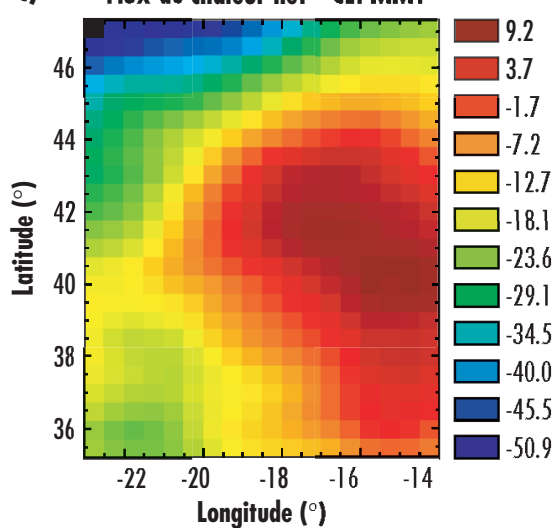

e) Vents CEPMMT ( $\mathrm{m} / \mathrm{s})$

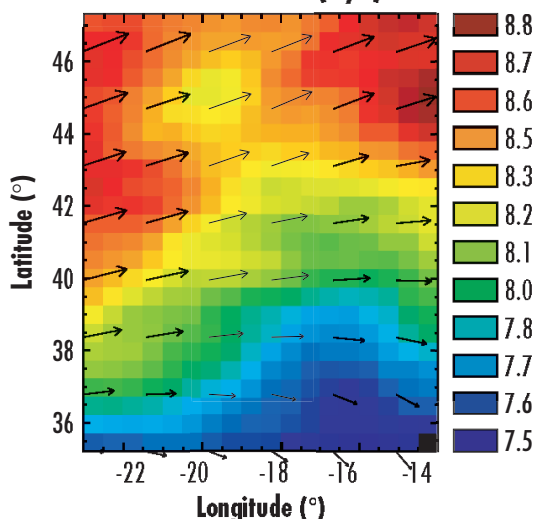

g) Vents SSM/I (m/s)

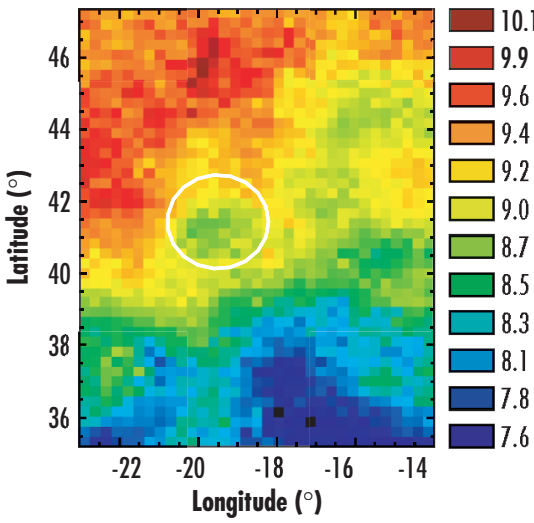

sité (Bourras et al., 2004). Sur la figure 3 , on note aussi l'écart conséquent entre flux obtenus par satellites et flux du CEPMMT, en valeur absolue. D'après les flux satellitaires, l'océan perd de la d) Flux de chaleur net - satellite $\left(\mathrm{W} / \mathrm{m}^{2}\right)$

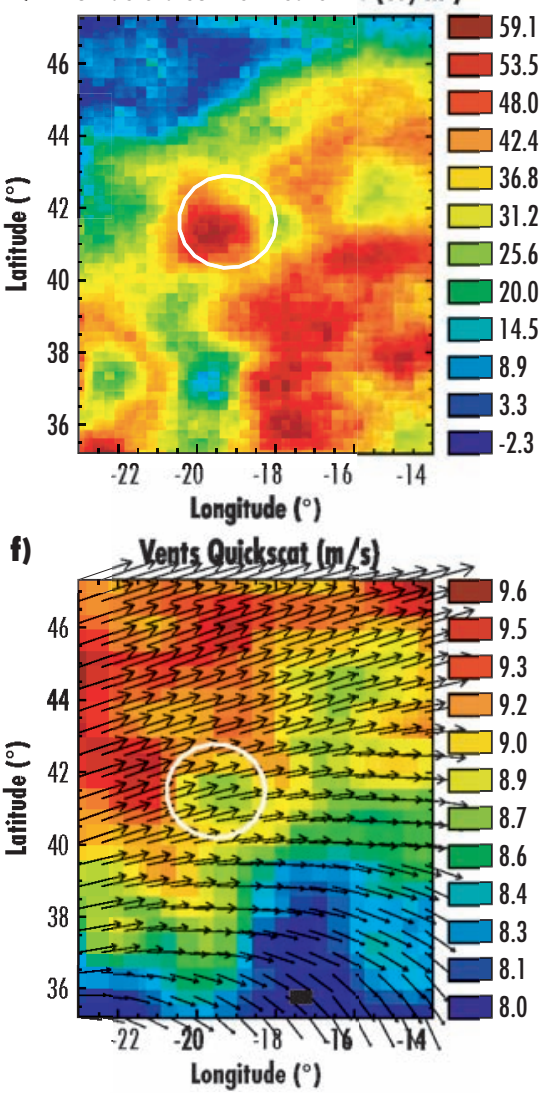

Figure 3 - Moyennes du 1er février au 30 avril 2001 sur la région Pomme, de la TSM $(\mathrm{a}, \mathrm{b})$, du vent, module et direction, représenté par les couleurs et les flèches (e,f.g) et du flux entrant dans l'océan $(c, d)$, d'après les analyses du CEPMMT $(a, c, e)$ et les observations spatiales $(b, d, f, g)$.

Les cercles en blanc repèrent le tourbillon froid (voir texte). Noter que les échelles de couleur sont différentes dans les champs $a, b$

les champs $\mathrm{c}, \mathrm{d}$ et e,f,g, de manière à mettre en évidence les variations spatiales des différentes variables. Pour le flux net, les valeurs

sont positives si l'océan perd de la chaleur (flux dirigé vers le haut,

c'est-à-dire de l'océan vers l'atmosphère). 
chaleur sur la majorité de la région pour les flux du CEPMMT. Cela illustre bien les incertitudes liées à la détermination des flux de surface, de l'ordre d'une trentaine de watts par mètre carré, en particulier dans les régions des latitudes moyennes où les flux annuels s'annulent, comme c'est le cas pour Pomme (Caniaux et al., 2005). Les figures $3 \mathrm{f}$ et $3 \mathrm{~g}$ révèlent de nombreuses structures de moyenne échelle dans le champ de vent, dont une correspond en taille et en position au tourbillon froid. $\mathrm{Au}$ centre du tourbillon, le module du vent est plus faible de 0,7 à $0,9 \mathrm{~m} . \mathrm{s}^{-1}$ qu'à la périphérie du tourbillon.

Sur les anomalies, le lien entre vent et température est détectable à des échelreprésente une série temporelle de la TSM et des vents SSM/I et Quikscat le long d'une coupe de $22^{\circ} \mathrm{W}-40,5^{\circ} \mathrm{N}$ à $17^{\circ} \mathrm{W}-42,5^{\circ} \mathrm{N}$. On note que la TSM est remarquablement stationnaire tout au long de la POI, bien qu'elle se décale progressivement d'un degré de longitude vers l'ouest après le 10 avril (jour 100). Dans les séries temporelles du vent, on perçoit clairement une zone plus sombre indiquant le lien entre TSM et vent tout au long de la POI (figures Pomme, alors que c'est le contraire les inférieures à trois mois. La figure 4

$4 b$ et $4 c$ ). Le signal est bien sûr peu apparent dans les séries de vent, ce qui montre que l'écoulement synoptique prend souvent le dessus sur l'action du gradient de TSM, probablement parce que ce dernier est faible. À cet égard, on remarque que le ralentissement du vent au passage de l'anomalie de TSM n'est clairement relié ni à l'intensité ni à la direction du vent moyen sur la zone (figure 4a).

\section{Simulations numériques}

\section{Influence du tourbillon froid sur la couche limite atmosphérique}

La simulation numérique est un outil adapté pour établir clairement la relation de cause à effet entre tourbillon froid et affaiblissement du vent. Pour cette raison, nous avons conduit des tests de sensibilité de l'atmosphère à la TSM, grâce à un modèle non hydrostatique à mesoéchelle, MM5 (Dudhia, 1993). L'atmosphère de la région
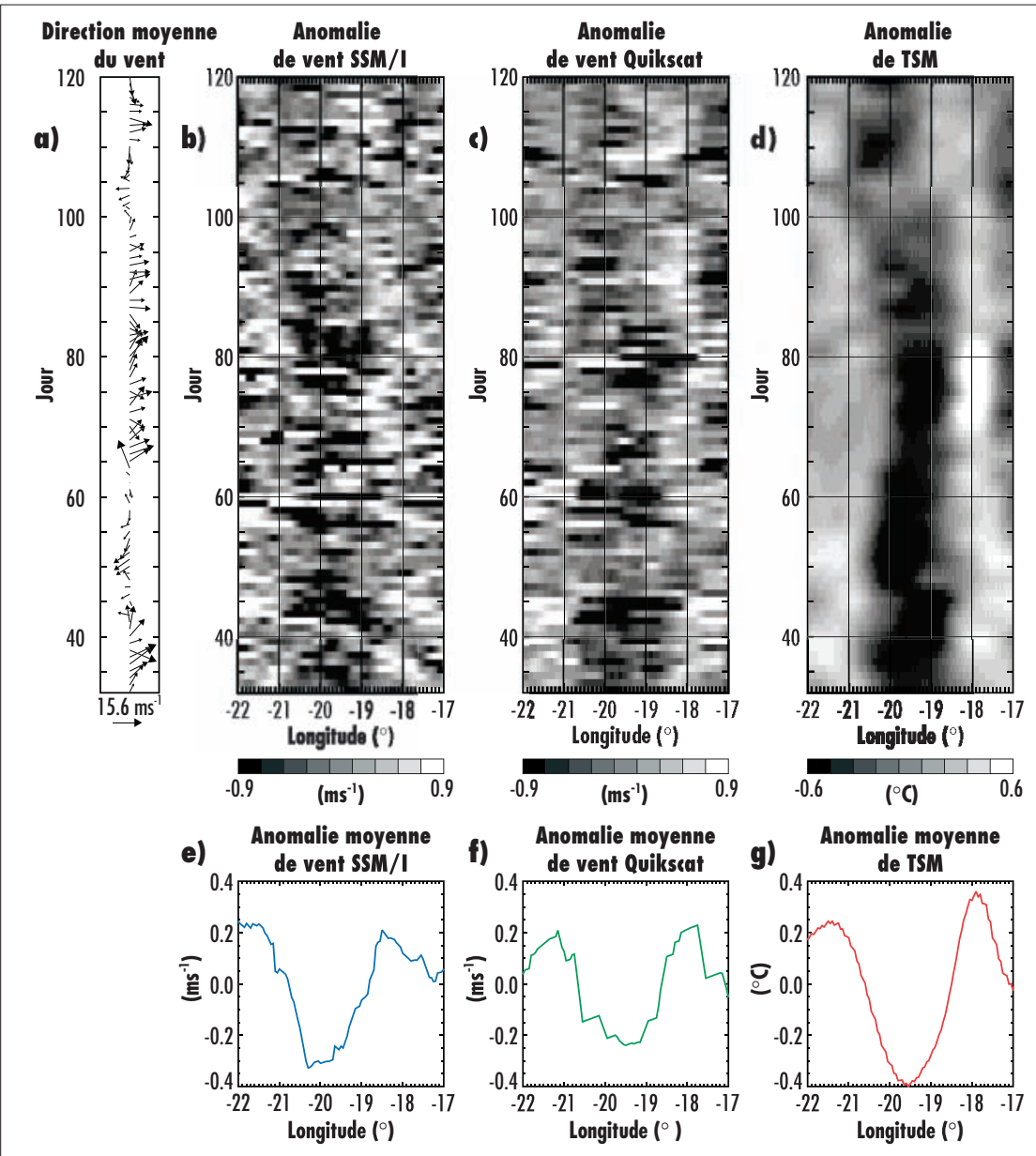

Pomme a fait l'objet de deux simulations, dans lesquelles la TSM provenait respectivement du CEPMMT (simulation de référence) et de Caniaux et al. (2005) (simulation réaliste). Les autres conditions aux limites et initiales provenaient du modèle du CEPMMT.

Cette stratégie permet d'étudier comment l'atmosphère se modifie lorsque le tourbillon froid est présent (simulation réaliste) ou non (simulation de référence) dans les champs de TSM. La figure 5 révèle que la signature du tourbillon froid est présente dans le champ de vent pour la simulation réaliste, tout comme dans les champs de vent satellitaires, alors qu'aucune anomalie de vent n'apparaît dans la simulation de référence. Cela permet d'établir avec certitude que la présence d'une zone de vent plus faible à l'emplacement du tourbillon froid est le fait de ce même tourbillon.

Nous n'avons constaté la présence d'aucune circulation secondaire, à partir des valeurs brutes des champs dynamiques. En effet, la dynamique atmosphérique est très semblable pour la simulation de référence et pour la simulation réaliste. En revanche, lorsque l'on s'intéresse à l'anomalie de circulation entre simulation réaliste et simulation de référence, on note clairement la présence d'une cellule de vent liée aux effets de surface, représentée figure 6 .

En anomalie par rapport à la simulation de référence,

- cette circulation secondaire comporte une zone de subsidence au niveau du front de TSM qui se situe entre le tourbillon froid et un tourbillon chaud situé juste à sa droite sur la figure 6 ;

- l'écoulement est dirigé du front de TSM vers le tourbillon froid, ce qui signifie bien que le vent absolu ralentit au passage du tourbillon.

Il est important de préciser qu'il y a aussi une accélération du vent à la sortie du tourbillon, ce qui signifie que ce sont les gradients de TSM qui agissent sur le vent, et non le tourbillon lui-même. En altitude, le phénomène s'inverse, et l'écoulement s'accélère à la verticale du tourbillon froid. Enfin, on constate que

Figure 4 - Évolution temporelle de la direction et du module du vent

(en anomalie par rapport au vent moyen), déduites d'observations spatiales (a-c) et évolution de l'anomalie de TSM (d), le long d'une coupe de 22W-40,5N à 17W-42,5N. Les profils moyens de vent

et de TSM associés sont présentés en e-g. 
Figure 5 - Moyennes des vents de surface durant la POI, d'après la simulation de référence (a) et la simulation réaliste (b) Le cercle blanc repère la position du tourbillon froid.

la circulation atteint le sommet de la couche limite. Cependant, la circulation est surtout perturbée par la surface dans les 200 premiers mètres. Cela est en très bon accord avec les résultats de Giordani et Planton (2000). Ces auteurs ont constaté qu'une couche limite interne se formait au-dessus d'un gradient faible de TSM, c'est-à-dire dans les 200 premiers mètres de 1'atmosphère.

\section{Processus responsables de la génération des circulations}

Grâce à un jeu d'équations diagnostiques établi par Sawyer (1956) et Eliassen (1962) pour des problèmes de frontogenèse, puis étendu à trois dimensions et adapté aux études proches de la surface par Giordani et Planton (2000), nous pouvons étudier quel processus est prépondérant dans
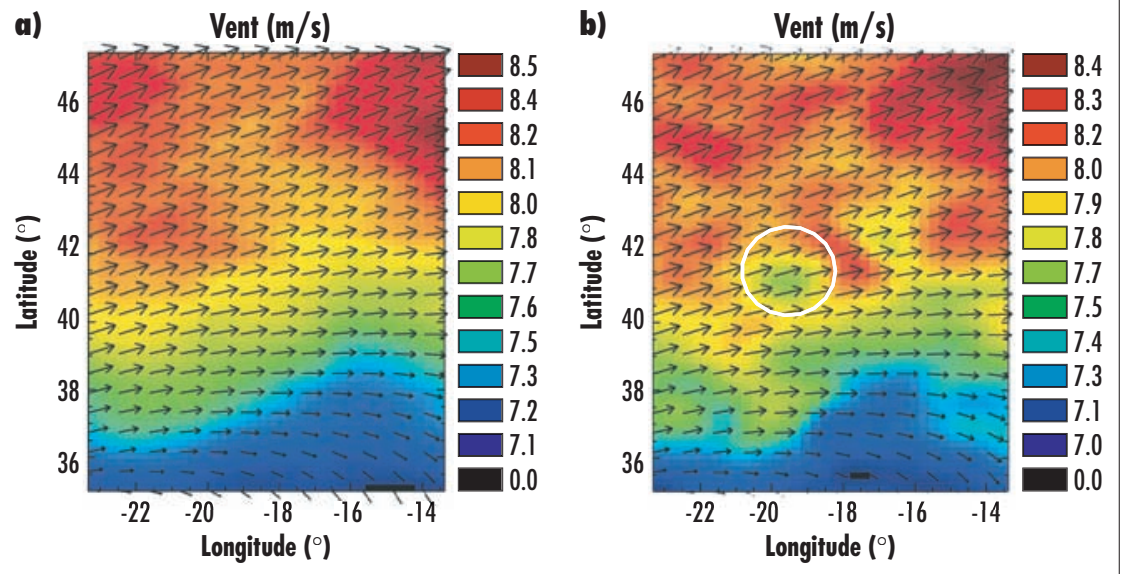

la génération des circulations secondaires à partir des simulations MM5. L'équation de Giordani et Planton (2000) décrit la circulation agéostrophique répondant à différents forçages, exprimés sous forme de cinq termes d'origine dynamique et thermique. Parmi ces différents forçages, on compte un terme lié aux effets de pression et un autre dû au frottement de l'écoulement atmosphérique contre la surface. Lorsque l'on applique un tel diagnostic aux simulations MM5 de Pomme, on montre que l'anomalie de vent agéostrophique est liée au forçage par le frottement du vent contre la surface : la turbulence est plus faible au-dessus du tourbillon froid car l'atmosphère y est naturellement plus stable, ce qui ralentit le vent, comme expliqué auparavant (voir Wallace et al., 1989, ou Hashizume et al., 2002, par exemple).

Le lecteur pourra trouver des compléments de détails de méthodologie, les équations, la démonstration des processus responsables de la réponse de l'atmosphère au tourbillon froid et les graphiques associés dans Bourras et al., (2004).

\section{Conclusions}

Nous avons présenté une étude de cas, dans laquelle un tourbillon cyclonique océanique, légèrement plus froid que son environnement, a influencé la couche limite atmosphérique en moyenne pendant trois mois. Lors de la campagne Pomme, les mesures effectuées en surface et la télédétection spatiale ont permis d'observer ce tourbillon, d'une centaine de kilomètres de diamètre. Ces mêmes données nous ont permis d'établir que le vent ralentissait d'environ $1 \mathrm{~m} / \mathrm{s}$ au passage du tourbillon froid, et que l'océan gagnait plus de chaleur au centre qu'à la périphérie du tourbillon. Le lien entre vent et TSM a été établi à échelle de trois mois, mais on a aussi montré qu'il existait à l'échelle journalière, bien que de manière épisodique, car il est masqué par le signal d'échelle synoptique du vent.

Un modèle météorologique a été utilisé pour mettre en évidence le lien de cause à effet entre les hétérogénéités de surface et la circulation atmosphérique. Il a également permis de visualiser des circulations secondaires dans le plan vertical, associées à ce forçage différentiel, en anomalie par rapport à une simulation de référence où le tourbillon n'était pas présent.

Enfin, nous avons pu montrer que le processus qui générait le ralentissement du vent était lié, non pas aux effets de pression, mais à celui de l'intensité du mélange turbulent, moins important au passage du tourbillon froid. b)

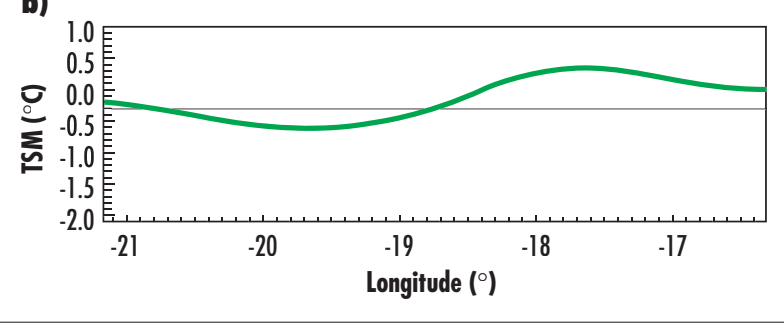

Figure 6 - Structures dynamique et thermodynamique de la couche limite atmosphérique dans le plan vertical, le long d'une coupe de 21,2W-41,5N à 16,2W-41,5N, d'après les simulations MM5.

La figure représente l'état de l'atmosphère relatif à la simulation de référence (simulation réaliste moins simulation de référence).

Le graphe b représente l'anomalie de TSM le long du plan de coupe.

La hauteur de couche limite est repérée par un trait tireté épais. Les contours montrent le champ d'anomalies de température positives en rouge trait plein, et négatives en rouge pointillé. 
Nos résultats montrent pour la première fois qu'un tourbillon faiblement énergétique a un impact sur la couche limite atmosphérique qui reste marqué quand on fait la moyenne sur plusieurs mois. Dans la mesure où il est possible de généraliser ce résultat à l'ensemble des océans où les gradients de TSM sont faibles, aux latitudes moyennes, nos résul- tats indiquent qu'il est nécessaire de prendre en compte ces tourbillons pour étudier la variabilité climatique, au moins aux échelles intrasaisonnières. En d'autres termes, il est indispensable de prendre en compte les effets de petite échelle de temps et d'espace, souvent qualifiés de « bruit » par les climatologues. Pour que cela soit possible, il serait utile d'ac- croître les moyens d'observation à haute résolution de l'océan et la résolution des modèles météorologiques (les tourbillons ne sont pas présents dans les champs de TSM utilisés par le CEPMMT). À défaut, une piste alternative serait de paramétrer les phénomènes de réponse de l'atmosphère aux hétérogénéités de surface dans les modèles de grande échelle.

\section{Bibliographie}

\footnotetext{
Bourras D., W. T. Liu, L. Eymard et W. Tang, 2003 : Evaluation of Latent Heat Flux Fields from Satellites and Models Over the Semaphore Region. J. Appl. Meteor., 42, 227-239

- Bourras D., G. Reverdin, H. Giordani et G. Caniaux, 2004 : Response of the atmospheric boundary layer to a mesoscale oceanic eddy in the northeast Atlantic, J. Geophys. Res., 109, D18114, doi:10.1029/2004JD004799.

- Caniaux G., A. Brut, D. Bourras, H. Giordani, A. Paci, L. Prieu et G. Reverdin, 2005 : A 1-Year Sea Surface Heat Budget in the NorthEastern Atlantic Basin During the - Pomme Experiment. 1. Flux estimates, J. Geophys. Res., 110, C07S02, doi:10.1029/2004JC002596.

Chelton D. B., S. K. Esbensen, M. G. Schlax, N. Thum, F. Wentz, C. L. Gentemann, M. J. McPhaden et P. S. Schopf, 2001 : Observation of Coupling between - Surface Wind Stress and Sea Surface Temperature in the Eastern Tropical Pacific, J. Climate, 14, 1479-1498.

- Ducet N., P. Y. Le Traon et G. Reverdin, 2000 : Global high-resolution mapping of ocean circulation from Topex/Poseidon and ERS-1 and -2, J. Geophys. Res., 105, 19477-19498.

- Dudhia J., 1993 : A Nonhydrostatic Version of the Penn State-NCAR Mesoscale Model: Validation Tests and Simulation of an Atlantic Cyclone and Cold Front, Mon. Wea. Rev., 121, 1493-1513

Eliassen A., 1962 : On the vertical circulation in frontal zones, Geophys. Norv., 24, 147-160.

Eymard L., S. Planton, P. Durand, C. Le Visage, P. Y. Le Traon, L. Prieur, A. Weill, D. Hauser, J. Rolland, J. Pelon, F. Baudin, B. Bénech, J. L. Bringuier, G. - Caniaux, P. De Mey, E. Dombrowski, A. Druilhet, H. Dupuis, B. Ferret, C. Flamant, F. Hernandez, D. Jourdan, K. Katsaros, D. Lambert, J. M. Levfevre, P. Le

- Borgne, B. Le Squere, A. Marsoin, H. Roquet, J. Tournadre, V. Trouillet, A. Tychensky et B. Zakardjian, 1996 : Study of the air-sea interactions at the mesoscale: - The Semaphore experiment, Ann. Geophys., 14, 986-1015.

Friehe C. A., W. J. Shaw, D. P. Rogers, K. L. Davidson, W. G. Large, S. A. Stage, G. H. Crescenti, S. J. S. Khalsa, G. K. Greenhut et F. Li, 1991 : Air-Sea Fluxes - and Surface Layer Turbulence Around a Sea Surface Temperature Front, J. Geophys. Res., 96, 8593-8609.

Giordani H., S. Planton, B. Bénech et B.-H. Kwon, 1998 : Atmospheric boundary layer response to sea surface temperatures during the Semaphore experiment, J. Geophys. Res., 103, 25047-25060.

- Giordani H. et S. Planton, 2000 : Modeling and Analysis of Ageostrophic Circulation over the Azores Oceanic Front during the Semaphore Experiment, Mon. Wea. Rev., 128, 2270-2287

Hashizume H., S.-P. Xie, M. Fujiwara, M. Shiotani, T. Watanabe, Y. Tanimoto, W. T. Liu et K. Takenchi, 2002 : Direct Observations of Atmospheric Boundary Layer - Response to SST Variations Associated with Tropical Instability Waves over the Eastern Equatorial Pacific, J. Climate, 15, 3379-3393.

Khalsa S. J. S. et G. K. Greenhut, 1989 : Atmospheric turbulence structure in the vicinity of an oceanic front, J. Atmos. Sci., 42, 1283-1305.

- Kwon B.-H., B. Bénech, D. Lambert, P. Durand, A. Druilhet, H. Giordani et S. Planton, 1998 : Structure of the marine atmospheric Boundary layer over an oceanic thermal front: Semaphore experiment, J. Geophys. Res., 103, 25159-25180.

Kilpatrick K.A., G. P. Podesta et R. Evanso, 2001 : Overview of the NOAA/Nasa Advanced Very High Resolution Radiometer Pathfinder algorithm for sea surface tem- perature and associated matchup database, Journal of Geophysical Research-Oceans, 106 C5, 9179-9197.

- Rogers D. P., 1989 : The Marine Boundary Layer in the Vicinity of an Ocean Front, J. Atmos. Sci., 46, 2044-2062.

Sawyer J. S., 1956 : The vertical circulation at meteorological fronts and its relation to frontogenesis, Proc. Roy. Soc. London, Ser. A., 234, 346-362.

- Stage S. A. et R. A. Weller, 1985 : The Frontal Air-Sea Interaction Experiment Fasinex. Part I: Background and Scientific Objectives, Bull. Amer. Meteor. Soc., 66, $1511-1520$.

- Simpson J. E., 1994 : Sea Breeze and Local Wind. Cambridge University Press, 234 p.

Wallace J. M., T. P. Mitchell et C. Deser, 1989 : The Influence of Sea-Surface Temperature on Surface Wind in the Eastern Equatorial Pacific: Seasonal and Interanual - Variability, J. Climate, 2, 1492-1499.

Warner T. T., M. N. Lakhtakia, J. D. Doyle et R. A. Pearson, 1990 : Marine atmospheric boundary layer circulations forced by Gulf Stream sea surface temperature - gradients, Mon. Wea. Rev., 118, 309-323.

- White W. B. et J. L. Annis, 2003 : Coupling of Extratropical Mesoscale Eddies in the Ocean to Westerly Winds in the Atmospheric Boundary Layer, J. Phys. Oceanogr., 33, 1095-1107

Woodruff S. D., R. J. Slutz, R. L. Jenne et P. M. Steurer, 1987 : A comprehensive ocean-atmosphere data set, Bull. Amer. Meteor. Soc., 68, 1239-1250.
} 